\title{
BMJ Open Information and communication technology-based interventions for suicide prevention implemented in clinical settings: a scoping review protocol
}

\author{
Hwayeon Danielle Shin (D) , ${ }^{1,2}$ Juveria Zaheer, ${ }^{3,4,5}$ Terri Rodak, $^{6}$ John Torous (D) , \\ Gillian Strudwick (1D ${ }^{2}$
}

To cite: Shin HD, Zaheer J, Rodak T, et al. Information and communication technologybased interventions for suicide prevention implemented in clinical settings: a scoping review protocol. BMJ Open 2022;12:e056232. doi:10.1136/ bmjopen-2021-056232

- Prepublication history and additional supplemental material for this paper are available online. To view these files, please visit the journal online (http://dx.doi.org/10.1136/ bmjopen-2021-056232)

Received 08 August 2021 Accepted 05 January 2022

Check for updates

(C) Author(s) (or their employer(s)) 2022. Re-use permitted under CC BY-NC. No commercial re-use. See rights and permissions. Published by BMJ.

For numbered affiliations see end of article.

Correspondence to

Ms Hwayeon Danielle Shin;

hdanielle.shin@mail.utoronto.ca

\section{ABSTRACT}

Introduction There is a surplus of information and communication technology (ICT)-based interventions for suicide prevention. However, it is unclear which of these ICT-based interventions for suicide prevention have been implemented in clinical settings. Furthermore, evidence shows that implementation strategies have often been mismatched to existing barriers. In response, the authors recognise the critical need for prospectively assessing the barriers and facilitators and then strategically developing implementation strategies. This review is part of a multiphase project to develop and test tailored implementation strategies for mobile app-based suicide prevention in clinical settings. The overall objective of this scoping review is to identify and characterise ICTbased interventions for all levels of suicide prevention in clinical settings. Additionally, this review will identify and characterise the barriers and facilitators to implementing these ICT-based interventions as well as reported measures and outcomes. The findings will directly inform the subsequent phase to maximise implementation and inform future efforts for implementing other types of ICTbased interventions related to suicide prevention in clinical settings.

Methods and analysis This review will adhere to the methods described by the Joanna Briggs Institute for conducting scoping reviews. The reporting will follow the Preferred Reporting Items for Systematic Reviews and Meta-Analyses extension for scoping review checklist. The following databases will be searched: Medline, Psyclnfo, Embase, Cumulative Index to Nursing \& Allied Health Literature (CINAHL), Web of Science and Library, Information Science \& Technology Abstracts (LISTA). Two reviewers will independently screen the articles and extract data using a standardised data collection tool. Then, authors will characterise extracted data using frameworks, typology and taxonomies to address the proposed review questions.

Ethics and dissemination Ethics approval is not required for this scoping review. Authors will share the results in a peer-reviewed, open access publication and conference presentations. Furthermore, the findings will be shared with relevant health organisations through lay language summaries and informal presentations.

\section{Strengths and limitations of this study}

- A rigorous scoping review method described by the Joanna Briggs Institute will be followed and the full report will be developed using the Preferred Reporting Items for Systematic Reviews and MetaAnalyses extension for scoping review checklist.

- This will be the first scoping review to map out the clinician-reported barriers and facilitators to implement ICT-based interventions for suicide prevention in clinical settings.

- This scoping review has limitations regarding the number of databases and non-English publication languages, which may limit research from lowincome and middle-income countries.

- The list of barriers and facilitators will be limited to what is reported in the existing literature; therefore, the research team aims to conduct a follow-up qualitative research to better investigate and contextualise barriers and facilitators in a specific clinical context.

- Since the purpose of this scoping review is to map and characterise the evidence, there will not be critical appraisals to determine the quality of individual studies to assess the risk of bias.

\section{INTRODUCTION}

Globally, 800000 people die by suicide every year, which translates to 11 deaths per day. ${ }^{1}$ In Canada, suicide is currently the second leading cause of death among youth and young adults. ${ }^{2}$ Furthermore, the service utilisation for those experiencing suicidal thoughts and behaviours has been increasing for many years. At a national level, the number of emergency department visits related to suicide-related thoughts and behaviours have doubled among youth between 2007 and 2015 in the USA. ${ }^{3}$ Similarly, in Australia, the numbers have tripled among patients of all ages from 2009 to 2018 in two emergency departments. ${ }^{4}$ Suicide prevention is a 
top research priority globally, as reflected in the United Nations Sustainable Development Goals for $2030 .^{5}$

The current COVID-19 pandemic has brought a significant impact on psychological health, further contributing to the increased need for suicide prevention services. ${ }^{6}$ Furthermore, there has been poorer access to mental health services since the onset of pandemic. ${ }^{7}$ One recommendation to meet this unprecedented, increased need for mental healthcare is to reform the system and redistribute services and resources from tertiary care centres to community and primary care. ${ }^{8}$ Technological integration between primary, community and tertiary mental health is one adaptable response. ${ }^{9}$ For example, innovative tools, such as telehealth tools, have been rapidly implemented in community mental health services internationally, allowing continuity of care. ${ }^{7}$ As such, COVID-19 pandemic has accelerated the implementation of digital solutions, ${ }^{10}$ and this momentum can be leveraged to redistribute mental healthcare via innovative means to provide appropriate suicide prevention care to patients at the right time.

Information and communication technology (ICT) is defined as 'a set of technologies resulting from the convergence of information technology and advanced multimedia and telecommunications techniques, which have enabled the emergence of more efficient means of communication, by improving processing, storage, distribution and exchange some information. ${ }^{11}$ ICTs are also referred to as eHealth by the $\mathrm{WHO},{ }^{12}$ and examples include, but are not limited to, internet and mobile technologies. There is a surplus of ICT-based interventions for mental healthcare, including suicide prevention. ${ }^{13}{ }^{14}$ For example, there are 38 mental health apps available from the Google Play Store (Android) and Apple App Store, and 11 of them are comprised of in-app crisis resources such as safety planning intervention (SPI) ${ }^{15}$ Rassy and colleagues ${ }^{14}$ have shown that ICTs for suicide prevention can provide an interactive, personalised and accessible way to reach various populations to identify and provide care to the individuals at risk. ${ }^{14}$ Although more additional higher quality, randomised controlled studies are required, evidence to date shows promising outcomes of the ICT-based interventions for suicide prevention, including high acceptability from the patients and some beneficial effects on suicidal ideation. ${ }^{16}$ In clinical settings, on the other hand, it remains unknown which ICT-based interventions for suicide prevention have been implemented and are being delivered by clinicians.

As of now, the $\mathrm{SPI}^{17}$ is one best practice for suicide prevention, producing a $45 \%$ reduction in suicidal behaviour compared with treatment as usual in the emergency department. ${ }^{18}$ More recently, systematic reviews have shown significant effects of SPI on reducing the risk of suicide-related behaviours. ${ }^{19}{ }^{20}$ SPI is a collaborative process between clinicians and a patient for developing a plan regarding coping strategies, emergency contacts and lethal means restriction. ${ }^{17}$ As collaboration is a critical feature of SPI, clinicians play an important role in creating a therapeutic alliance with patients and building trust. As such, ICTs cannot replace clinicians in situations where clinical interaction is essential, yet ICTs can be embedded in clinical settings to make effective interventions more widely accessible. ${ }^{21}$ Furthermore, integrating ICT-based interventions into routine care, which allows clinicians to provide oversight to patients, can facilitate the adoption of these tools. ${ }^{22}$

To date, reviews have yet to comprehensively explore ICT-based interventions for suicide prevention delivered in clinical settings. Furthermore, reviews have yet to assess barriers and facilitators for implementing these ICT-based interventions for suicide prevention. Hence, the overall objective of this scoping review is to identify and characterise ICT-based interventions for all levels of suicide prevention in clinical settings. The secondary objectives of this review are as follow: (1) identify and characterise the barriers and facilitators to implementing these ICT-based interventions within the capability, opportunity, motivation-behaviour (COM-B $)^{23}$ and the Theoretical Domains Framework (TDF) ${ }^{24}$ and (2) identify reported measures and outcomes in these studies.

This review is part of a multiphase project to develop and test tailored implementation strategies for mobile appbased suicide prevention in clinical settings. Implementation is a known determinant of effectiveness, meaning barriers to implementation can significantly reduce the effectiveness of interventions and lead to suboptimal outcomes. ${ }^{25}$ Furthermore, evidence shows that implementation strategies have often been mismatched to existing barriers in a given context. ${ }^{26}{ }^{27}$ For example, a review of 20 quality improvement studies found that many studies used clinician-oriented (individual level) strategies, such as education, to address organisational level barriers. ${ }^{26}$ Theoretically speaking, education alone may be insufficient to address external influences of implementation. ${ }^{23}$ Specifically for mental health apps, there was a recent call for attention to complex contexts in which apps are being implemented in order to tailor facilitation. ${ }^{28}$ As such, it is critical to prospectively assess the barriers and facilitators in the organisational and local context and then strategically develop implementation strategies. ${ }^{29}$ The findings from this review will be used as a knowledge base for the subsequent phase to identify strategies to overcome barriers and leverage facilitators to maximise implementation. The findings can also inform future efforts to develop and test strategies for implementing other types of ICT-based interventions related to suicide prevention in clinical settings.

A search of PROSPERO, the Cochrane Database of Systematic Reviews and Joanna Briggs Institute (JBI) Evidence Synthesis and Open Science Framework was conducted in June-July 2021, and no current or underway systematic reviews or scoping reviews on the topic were identified. 


\section{RESEARCH QUESTIONS}

To achieve the research objectives stated above, this scoping review will address the following questions.

1. What ICT-based interventions for suicide prevention have been implemented in clinical settings?

1.1. What are the reported barriers and facilitators to implementing these ICT-based interventions?

1.2. What are the reported measures and outcomes?

\section{METHODS}

This review will adhere to the JBI methodology for scoping review, ${ }^{31}$ and the Preferred Reporting Items for Systematic Reviews and Meta-Analyses (PRISMA) extension for scoping reviews ${ }^{32}$ will be used to guide the reporting. Major steps of our scoping review are: (1) searching for relevant studies; (2) screening and selecting relevant studies; (3) extracting data and (4) summarising and presenting key findings.

\section{INCLUSION/EXCLUSION CRITERIA}

To identify relevant studies, key inclusion/exclusion criteria were constructed based on the population, concept and context mnemonic recommended by JBI.

\section{Participant}

In the literature, a wide range of healthcare professionals who provide direct care in clinical settings (eg, physicians, nurses, nurse practitioners, physician assistants, social workers and medical resident) have been commonly referred to as 'clinicians'. ${ }^{33}$ All types of clinicians who are licenced and regulated practitioners will be included in this review. Furthermore, unregulated practitioners or clinical support personnel, such as peer support workers, will be included in this review. Therefore, ICT-based interventions must be implemented or delivered by these members of the clinical team, and this review will exclude studies if a research assistant delivers an ICT-based intervention. There will be no exclusion criteria based on gender, healthcare discipline and years of experience. Therefore, healthcare trainees, such as medical residents, will also be considered for inclusion. Finally, ICT-based interventions can target patient population of any age and any levels of suicide prevention.

\section{Concept}

All literatures that describe ICT-based interventions will be included in this review. Following the definitions provided in the introduction, ICTs or eHealth include, but are not limited to, computerised resources, mobile apps and text messaging. Additionally, the definition provided by the WHO will be adopted to identify interventions: 'A health intervention is an act performed for, with or on behalf of a person or population whose purpose is to assess, improve, maintain, promote or modify health, functioning or health conditions. ${ }^{35}$ Therefore, general use of electronic healthcare records while caring for patients with suicidal ideation will be excluded if there are no ICT-based interventions being implemented or delivered to patients. Additionally, routine care (ie, care as usual) provided via virtual platforms or telephones will be excluded unless an ICT-based intervention is being delivered. ICT-based interventions can be delivered in-person or other means of communication by clinicians. Crisis services (phone, chat, text) are appropriate response for suicide prevention. However, this review will exclude crisis services since there has already been a systematic review investigating their effectiveness. ${ }^{36}$ The current review will use the umbrella term, suicide-related thoughts and behaviours, ${ }^{37}$ which refers to a spectrum of suicide-related ideation, communication, behaviours and attempts with having casual to persistent suicidal thoughts with actual, undetermined or no suicidal intent. ICT-based interventions for suicide prevention regarding any subcategory of suicide-related thoughts and behaviours will be included. Finally, ICT-based interventions related to all levels of suicide prevention following the WHO description (ie, Universal, Selective, Indicated) ${ }^{38}$ will be included. See table 1 for the list of suicide prevention interventions.

\section{Context}

All types of clinical settings, such as in-patient, emergency, ambulatory and primary care, will be considered for inclusion. For this review, a clinical setting is defined as any context where clinician-patient interactions occur in real time. To be included, ICT-based interventions need to be implemented and initiated in the clinical setting. Self-support tools that patients can freely download from app stores will be excluded as these are being initiated in non-clinical settings. However, if app-based interventions or other forms of ICT-based interventions are prescribed by clinicians or clinical support team, they will be included in the review. Please see table 1 for the summary of eligibility.

\section{SEARCH STRATEGY}

In collaboration with a health science librarian, a comprehensive search strategy will be developed to locate relevant scholarly literature using multiple bibliographic databases. This scoping review will follow a three-step search strategy outlined in JBI methodology. ${ }^{31}$ First, we will develop and refine a draft strategy in Medline, followed by an analysis of the text words contained in titles and abstracts of relevant articles and the subject headings applied to them. After revising, testing and finalising this search strategy, TR will translate the strategy using database-specific subject headings, search fields and operators and run the search in each included database. The search strategy will be peer reviewed by a second research librarian using the Peer Review of Electronic Search Strategy guidelines. ${ }^{39}$ The proposed search strategy for Medline (Ovid), peer-reviewed by a second research librarian, is presented in online supplemental file I. Third, the reference list of the sources that have 


\begin{tabular}{|c|c|c|}
\hline & Inclusion criteria & Exclusion criteria \\
\hline Population & $\begin{array}{l}\text { All members of clinical care team } \\
\text { Licenced and regulated practitioners } \\
\text { Unregulated practitioners or clinical support teams such as peer support } \\
\text { workers } \\
\text { All ages, genders, locations and years of experience }\end{array}$ & $\begin{array}{l}\text { Not a member of clinical care } \\
\text { team }\end{array}$ \\
\hline
\end{tabular}

\begin{tabular}{lll} 
Setting & Clinical/hospital setting or context (ie, clinician-patient interaction in real & Not a clinical setting or context \\
time) & $\begin{array}{l}\text { Primary research papers including in press papers. If literature reviews, } \\
\text { commentaries and opinion papers include relevant primary research studies, opinion papers, literature reviews, } \\
\text { this review will include them in the screening phase then hand-search their } \\
\text { references to identify the original papers that meet the inclusion criteria. }\end{array}$ & $\begin{array}{l}\text { Books, theses, commentaries, } \\
\text { premstracts }\end{array}$ \\
& Conference papers, reports from relevant health services organisations. & N/A \\
Type of study & All designs including study protocols & non-English language \\
\hline Language & English &
\end{tabular}

been included in the reviews will be hand searched for additional articles.

The targeted Google search method outlined by Godin et $a l^{40}$ will be used to identify a list of international and national health services' websites to locate reports and other eligible sources. The first step involves conducting 10 unique Google searches with different combinations of keywords and reviewing the first 100 items of each search to identify relevant websites. The second step involves hand-searching-identified relevant websites to find reports or other sources that meet the inclusion criteria. This targeted Google search will complement the database searches to identify more diverse sources of evidence.

\section{TYPES OF SOURCES}

The following databases will be searched for relevant studies: Medline, APA PsycInfo, Embase, the Cumulative Index to Nursing \& Allied Health Literature (CINAHL), Web of Science and Library, Information Science \& Technology Abstracts (LISTA). All types of research designs will be included (eg, quantitative, observational, qualitative and mixed methods). Although study protocols do not have empirical data, we will include them and capture relevant details and reflect the upcoming trends. By doing so, we will be able to provide a comprehensive breadth of information that is currently available. Reference lists of relevant literature reviews, commentaries, text and opinion papers will be reviewed to identify additional primary research papers that meet the eligibility criteria. Grey literature including conference papers, reports and publications by relevant national and international websites of health organisations and agencies will be included. Sources written in English will be included, and no date parameters will be applied.

\section{STUDY SELECTION}

All identified citations will be collated and uploaded into Covidence ${ }^{41}$ and duplicates will be automatically removed. Two reviewers will independently screen titles and abstracts against the inclusion and exclusion criteria. Next, relevant full-text articles will be retrieved into 
Covidence, ${ }^{41}$ and two independent reviewers will assess the full text of relevant studies in detail against the eligibility criteria. Reasons for exclusion will be recorded at the full-text screening phase and will be reported in the full review. Any discrepancies between the reviewers at each stage of the study selection process will be resolved either through discussion or by a third reviewer. Scoping reviews do not require methodological assessment, ${ }^{31}$ so critical appraisal will not be conducted. The results of the study selection will be reported and presented in a PRISMA 2020 flow diagram. ${ }^{42}$

\section{DATA EXTRACTION}

Two reviewers will independently extract and chart data. The data extraction tool will be pilot tested with five studies to ensure consistency and assess the need for modification of the tool. Any modification of the tool will be reported in the full report. See online supplemental file II for the draft version of data extraction tool. Data will be extracted by two independent reviewers to capture the following information: general characteristics of the paper (title, year, author, country of origin and design), description and characteristics of clinical settings, geography, characteristics of participating clinicians, description and characteristics of implementation strategies and ICT-based intervention(s), target patient population, clinician-reported barriers and facilitators to implementing ICT-based interventions, reported measures, outcomes and direction of effectiveness. Any discrepancies in data extraction will be resolved either through discussion between the two reviewers or by a third reviewer. Finally, authors will be contacted to request for missing or additional information when appropriate.

\section{DATA ANALYSIS}

Following data extraction, this review will characterise extracted data using frameworks, typology and taxonomies to address the proposed review questions. Data coding strategy will be pilot tested and assessed for further modification. After finalising the coding strategy, the primary reviewer will code the rest of the data, and then the second reviewer will verify the coded data. Any disagreements that arise between the reviewers will be resolved either through discussion or by a third reviewer. Data coding is expected to be an iterative process; therefore, any necessary changes to the coding strategy will be made and reported in the full review.

\section{Characteristics of ICT-based interventions (question 1)}

Identified ICT-based interventions will be categorised using typology created by the Mental Health Commission of Canada. ${ }^{43}$ Then interventions will be categorised into the three levels of suicide prevention following the descriptions provided by the WHO: ${ }^{38}$ (1) Universal (entire population), (2) selective (specific subpopulations, targeting vulnerable populations), (3) indicated (high-risk individuals, displaying signs of suicide potential).

\section{Barriers and facilitators to implementing ICT-based interventions in clinical settings (question 1.1)}

This review will perform directed content analysis ${ }^{44}$ to describe clinician-reported barriers and facilitators to implementing ICT-based interventions within the COM-B and TDF. This review is part of a multiphase project to develop and test tailored implementation strategies for a mobile app-based suicide prevention in a clinical setting. Lynch and colleagues ${ }^{45}$ advice on selecting theory for implementation projects and suggest the use of COM-B and TDF when researchers are investigating individual experiences as a preparation for implementation. As such, the authors made evidence-informed decision to use COM-B and TDF. Both COM-B ${ }^{23}$ and TDF $^{24}$ are comprehensive and evidence-based behaviour frameworks that capture internal and external influences on an individual's behaviour change. Furthermore, COM-B and TDF have been previously used across healthcare disciplines to assess implementation problems and to provide theoryinformed suggestions for implementation. ${ }^{46-48}$ In the current scoping review, narrative descriptions of barriers and facilitators will be coded onto the most appropriate domains of COM-B and TDF. Coded barriers and facilitators will serve as a knowledge base in future research, informing the strategic selection of theory-based strategies for implementation that can overcome barriers and leverage facilitators.

\section{Reported measures and outcomes (question 1.2)}

This review will categorise reported measures and outcomes using the Effective Practice and Organisation of Care taxonomy ${ }^{49}$ and outcomes of evidence-based practice measures. ${ }^{50}$ Outcomes will be categorised into three levels: (1) patient, (2) healthcare provider and (3) health system. Patient-level outcomes will be further distinguished to patient-reported outcomes (eg, symptoms), ${ }^{51}$ patient-reported experience (eg, satisfaction) ${ }^{52}$ and patient health outcomes (eg, mortality). ${ }^{49}$ Examples of healthcare provider outcomes include knowledge, attitude (eg, satisfaction and acceptability) and behaviour (eg, practice changes noted in medical charts). ${ }^{50}$ Examples of system-level outcomes include resource utilisation (eg, length of stay and number of admission) and economic outcomes (eg, cost-effectiveness). ${ }^{49}$

\section{DATA PRESENTATION}

This review will present the charted data in tables that align with the review objectives. Descriptive numerical summaries of the quantitative data (eg, frequency counts for barriers and facilitators) will be provided where possible. Finally, narrative descriptions will accompany these presentations and describe how the findings address the review questions. 


\section{PUBLIC AND PATIENT INVOLVEMENT}

The authors plan to discuss the review findings and request feedback from the Suicide Prevention Working Group and the clinical patient and family advisory committees at the Centre for Additions and Mental Health (CAMH) for the next step of this work. During engagement meetings, these groups can identify research priorities to inform the next steps. Also, these groups will be invited to contribute to the dissemination plan.

\section{ETHICS AND DISSEMINATION}

This scoping review is aimed at synthesising information from the existing literature; therefore, ethics approval is not required. This scoping review is part of a multiphase project to develop and test tailored implementation strategies for a mobile app-based suicide prevention intervention in a clinical setting. The findings will directly inform the subsequent phase to identify strategies to overcome barriers and leverage facilitators to maximise implementation. Furthermore, authors anticipate that the findings will inform future research directions for other ICT implementation efforts in clinical settings. As such, authors will share the results in a peer-reviewed, open access publication and conference presentations. Furthermore, the findings will be shared with relevant health organisations (eg, CAMH) through lay language summaries and informal presentations.

\section{Author affiliations}

${ }^{1}$ Institute of Health Policy, Management and Evaluation, University of Toronto, Toronto, Ontario, Canada

${ }^{2}$ Campbell Family Mental Health Research Institute, Centre for Addiction and Mental Health, Toronto, Ontario, Canada

${ }^{3}$ Department of Psychiatry, University of Toronto, Toronto, Ontario, Canada

${ }^{4}$ Health Outcomes and Performance Evaluation (HOPE) Research Unit, Institute for Mental Health Policy Research, Centre for Addiction and Mental Health, Toronto, Ontario, Canada

${ }^{5}$ Gerald Sheff and Shanitha Kachan Emergency Department, Centre for Addiction and Mental Health, Toronto, Ontario, Canada

${ }^{6}$ Library, Centre for Addiction and Mental Health, Toronto, Ontario, Canada ${ }^{7}$ Department of Psychiatry, Beth Israel Deaconess Medical Center, Harvard Medical School, Boston, Massachusetts, USA

\section{Twitter Juveria Zaheer @juveriazaheer}

Contributors HDS designed the scoping review protocol including data collection and interpretation planning. HDS drafted the protocol. TR is a librarian and revised methods for search strategy and developed the final search strategy. HDS, JZ, TR, JT, GS revised the protocol for intellectual content and made a final approval for the submission of the protocol.

Funding The authors have not declared a specific grant for this research from any funding agency in the public, commercial or not-for-profit sectors.

Competing interests None declared.

Patient consent for publication Not applicable.

Provenance and peer review Not commissioned; externally peer reviewed.

Supplemental material This content has been supplied by the author(s). It has not been vetted by BMJ Publishing Group Limited (BMJ) and may not have been peer-reviewed. Any opinions or recommendations discussed are solely those of the author(s) and are not endorsed by BMJ. BMJ disclaims all liability and responsibility arising from any reliance placed on the content. Where the content includes any translated material, BMJ does not warrant the accuracy and reliability of the translations (including but not limited to local regulations, clinical guidelines, terminology, drug names and drug dosages), and is not responsible for any error and/or omissions arising from translation and adaptation or otherwise.

Open access This is an open access article distributed in accordance with the Creative Commons Attribution Non Commercial (CC BY-NC 4.0) license, which permits others to distribute, remix, adapt, build upon this work non-commercially, and license their derivative works on different terms, provided the original work is properly cited, appropriate credit is given, any changes made indicated, and the use is non-commercial. See: http://creativecommons.org/licenses/by-nc/4.0/.

\section{ORCID iDs}

Hwayeon Danielle Shin http://orcid.org/0000-0003-4037-4464

John Torous http://orcid.org/0000-0002-5362-7937

Gillian Strudwick http://orcid.org/0000-0002-1080-7372

\section{REFERENCES}

1 WHO. Suicide, 2019. Available: http://www.who.int/mental_health/ prevention/suicide/suicideprevent/en/ [Accessed 14 Mar 2021].

2 Public Health Agency of Canada. Suicide in Canada: Key Statistics (infographic) [Internet]. Government of Canada, 2019. Available: https://www.canada.ca/en/public-health/services/publications/ healthy-living/suicide-canada-key-statistics-infographic.html [Accessed 28 Sep 2019].

3 Burstein B, Agostino H, Greenfield B. Suicidal attempts and ideation among children and adolescents in US emergency departments, 2007-2015. JAMA Pediatr 2019;173:598-600.

4 Stapelberg NJC, Sveticic J, Hughes I, et al. Suicidal presentations to emergency departments in a large Australian public health service over 10 years. Int J Environ Res Public Health 2020;17. doi:10.3390/ ijerph17165920. [Epub ahead of print: 1408 2020].

5 WHO. Live life: preventing suicide, 2018. Available: https://cdn. who.int/media/docs/default-source/mental-health/suicide/live-lifebrochure.pdf?sfvrsn=6ea28a12_2\&download=true [Accessed 18 May 2021].

6 Sher $\mathrm{L}$. The impact of the COVID-19 pandemic on suicide rates. QJM Int J Med 2020;113:707-12. doi:10.1093/qjmed/hcaa202

7 Sheridan Rains L, Johnson S, Barnett P, et al. Early impacts of the COVID-19 pandemic on mental health care and on people with mental health conditions: framework synthesis of international experiences and responses. Soc Psychiatry Psychiatr Epidemiol 2021;56:13-24.

8 Xiang Y-T, Zhao Y-J, Liu Z-H, et al. The COVID-19 outbreak and psychiatric hospitals in China: managing challenges through mental health service reform. Int J Biol Sci 2020;16:1741-4.

9 Rosenberg S, Mendoza J, Tabatabaei-Jafari H, et al. International experiences of the active period of COVID-19 - Mental health care. Health Policy Technol 2020;9:503-9.

10 Golinelli D, Boetto E, Carullo G, et al. Adoption of digital technologies in health care during the COVID-19 pandemic: systematic review of early scientific literature. J Med Internet Res 2020;22:e22280.

11 The large terminological dictionary (GDT). technologies de l'information et de la communication [Internet]. Office québécois de la langue française. Available: http://gdt.oqlf.gouv.qc.ca/ficheOqlf. aspx?ld_Fiche $=8349341$ [Accessed 25 May 2021].

12 WHO. Frequently asked questions on Global Task Force on digital health for TB and its work [Internet]. WHO. World Health Organization. Available: http://www.who.int/tb/areas-of-work/digitalhealth/faq/en/ [Accessed 25 May 2021].

13 Larsen ME, Nicholas J, Christensen H. A systematic assessment of smartphone tools for suicide prevention. PLoS One 2016;11:e0152285.

14 Rassy J, Bardon C, Dargis L, et al. Information and communication technology use in suicide prevention: Scoping review. J Med Internet Res 2021;23:e25288.

15 Parrish EM, Filip TF, Torous J, et al. Are mental health Apps adequately equipped to handle users in crisis? Crisis 2021. doi:10.1027/0227-5910/a000785. [Epub ahead of print: 27 May 2021].

16 Arshad U, Ain F-U, Gauntlett J. A systematic review of the evidence supporting Mobile- and Internet-based psychological interventions for self-harm. Suicide Life Threat Behav 2020;50:151-79.

17 Stanley B, Brown GK. Safety planning intervention: a brief intervention to mitigate suicide risk. Cogn Behav Pract 2012;19:256-64.

18 Stanley B, Brown GK, Brenner LA, et al. Comparison of the safety planning intervention with follow-up vs usual care of suicidal patients treated in the emergency department. JAMA Psychiatry 2018;75:894-900. 
19 Nuij C, van Ballegooijen W, de Beurs D. Safety planning-type interventions for suicide prevention: meta-analysis. $\mathrm{Br} J$ Psychiatry 2021:1-8.

20 Ferguson M, Rhodes K, Loughhead M, et al. The effectiveness of the safety planning intervention for adults experiencing suicide-related distress: a systematic review. Arch Suicide Res 2021:1-24.

21 Bennett CB, Ruggero CJ, Sever AC, et al. eHealth to redress psychotherapy access barriers both new and old: a review of reviews and meta-analyses. J Psychother Integr 2020;30:188-207.

22 Schreiweis B, Pobiruchin M, Strotbaum V, et al. Barriers and facilitators to the implementation of eHealth services: systematic literature analysis. J Med Internet Res 2019;21:e14197.

23 Michie S, van Stralen MM, West R. The behaviour change wheel: a new method for characterising and designing behaviour change interventions. Implement Sci 2011;6:42.

24 Cane J, O'Connor D, Michie S. Validation of the theoretical domains framework for use in behaviour change and implementation research. Implement Sci 2012;7:37.

25 Proctor E, Silmere H, Raghavan R, et al. Outcomes for implementation research: conceptual distinctions, measurement challenges, and research agenda. Adm Policy Ment Health 2011;38:65-76.

26 Bosch M, van der Weijden T, Wensing M, et al. Tailoring quality improvement interventions to identified barriers: a multiple case analysis. J Eval Clin Pract 2007;13:161-8.

27 Wensing M, Grol R. Knowledge translation in health: how implementation science could contribute more. BMC Med 2019;17:88.

28 Connolly SL, Hogan TP, Shimada SL, et al. Leveraging implementation science to understand factors influencing sustained use of mental health Apps: a narrative review. J Technol Behav Sci 2020:1-13.

29 Lattie EG, Nicholas J, Knapp AA, et al. Opportunities for and tensions surrounding the use of Technology-Enabled mental health services in community mental health care. Adm Policy Ment Health 2020:47:138-49.

30 Peters M, Godfrey C, Mclnerney P. Chapter 11: Scoping Reviews. In: Aromataris E, Munn Z, eds. Joanna Briggs Institute Reviewer's Manual. The Joanna Briggs Institute, 2017. https://reviewersmanual. joannabriggs.org/

31 Peters MDJ, Marnie C, Tricco AC, et al. Updated methodological guidance for the conduct of scoping reviews. JBI Evid Synth 2020;18:2119-26.

32 Tricco AC, Lillie E, Zarin W, et al. PRISMA extension for scoping reviews (PRISMA-ScR): checklist and explanation. Ann Intern Med 2018;169:467-73.

33 Im DD, Chary A, Condella AL, et al. Emergency department clinicians' attitudes toward opioid use disorder and emergency Department-initiated buprenorphine treatment: a mixed-methods study. West J Emerg Med 2020;21:261-71.

34 Jelinek GA, Marck CH, Weiland TJ, et al. Organ and tissue donationrelated attitudes, education and practices of emergency department clinicians in Australia. Emerg Med Australas 2012;24:244-50.

35 WHO. International classification of functioning, disability and health (ICF). Available: https://www.who.int/standards/classifications/internationalclassification-of-health-interventions [Accessed 25 May 2021].

36 Hoffberg AS, Stearns-Yoder KA, Brenner LA. The effectiveness of crisis line services: a systematic review. Front Public Health 2019;7:399.

37 Silverman MM, Berman AL, Sanddal ND, et al. Rebuilding the Tower of Babel: a revised nomenclature for the study of suicide and suicidal behaviors. Part 2: suicide-related ideations, communications, and behaviors. Suicide Life Threat Behav 2007;37:264-77.

38 WHO. Preventing suicide: A global imperative [Internet]. World Health Organization, 2014. Available: https://www.who.int/publications/i/ item/9789241564779 [Accessed 23 Nov 2019].
39 McGowan J, Sampson M, Salzwedel DM, et al. PRESS Peer Review of Electronic Search Strategies: 2015 Guideline Statement. J Clin Epidemiol 2016;75:40-6.

40 Godin K, Stapleton J, Kirkpatrick SI, et al. Applying systematic review search methods to the grey literature: a case study examining guidelines for school-based breakfast programs in Canada. Syst Rev 2015;4:138.

41 Covidence systematic review software [Internet]. Melbourne, Australia, 2019. Available: www.covidence.org

42 Page MJ, McKenzie JE, Bossuyt PM, et al. The PRISMA 2020 statement: an updated guideline for reporting systematic reviews. BMJ 2021;372:n71.

43 Mental Health Commission of Canada. E-Mental Health in Canada: Transforming the Mental Health System Using Technology [Internet]. Ottawa, ON, 2014. Available: https://www.mentalhealthcommission. $\mathrm{ca} /$ sites/default/files/MHCC_E-Mental_Health-Briefing_Document_ ENG_0.pdf

44 Hsieh H-F, Shannon SE. Three approaches to qualitative content analysis. Qual Health Res 2005;15:1277-88.

45 Lynch EA, Mudge A, Knowles S, et al. "There is nothing so practical as a good theory": a pragmatic guide for selecting theoretical approaches for implementation projects. BMC Health Serv Res 2018;18:857.

46 Jabbour M, Newton AS, Johnson D, et al. Defining barriers and enablers for clinical pathway implementation in complex clinical settings. Implement Sci 2018:13:139.

47 Surr CA, Parveen S, Smith SJ, et al. The barriers and facilitators to implementing dementia education and training in health and social care services: a mixed-methods study. BMC Health Serv Res 2020;20:512

48 Glowacki K, Weatherson K, Faulkner G. Barriers and facilitators to health care providers' promotion of physical activity for individuals with mental illness: A scoping review. Ment Health Phys Act 2019:16:152-68.

49 Cochrane Effective Practice and Organisation of Care (EPOC). What outcomes should be reported in Cochrane effective practice and organisation of care (EPOC) reviews? EPOC resources for review authors. EPOC resources for review authors, 2017. Available: https:// epoc.cochrane.org/sites/epoc.cochrane.org/files/public/uploads/ Resources-for-authors2017/what_outcomes_should_be_reported_in epoc_reviews.pdf

50 Graham I, Bick D, Tetroe J. Measuring outcomes of evidence-based practice: Distinguishing between knowledge use and its impact. In: Bick D, Graham I, eds. Evaluating the impact of implementing evidence based practice. Chichester: John Wiley \& Sons, 2010: 18-37.

51 FDA. Guidance for Industry: Patient-Reported Outcome Measures: Use in Medical Product Development to Support Labeling Claims. [Internet]. Rockville, MD, 2009. Available: https://www.fda.gov/ regulatory-information/search-fda-guidance-documents/patientreported-outcome-measures-use-medical-product-developmentsupport-labeling-claims [Accessed 7 Dec 2020].

52 Kingsley C, Patel S. Patient-Reported outcome measures and patient-reported experience measures. BJA Educ 2017;17:137-44.

53 Wilson MP, Moutier C, Wolf L. Emergency department recommendations for suicide prevention in adults: the ICARE mnemonic and a systematic review of the literature. Am J Emerg Med 2019:38.

54 Zalsman G, Hawton K, Wasserman D, et al. Suicide prevention strategies revisited: 10-year systematic review. Lancet Psychiatry 2016;3:646-59. 